\title{
OPEN Multipartite uncertainty relation with quantum memory
}

\author{
Saeed Haddadi ${ }^{1,2 \bowtie}$, Mohammad Reza Pourkarimi ${ }^{3}{ }^{3}$ \& Soroush Haseli ${ }^{4}$
}

We present a new quantum-memory-assisted entropic uncertainty relation for multipartite systems which shows the uncertainty principle of quantum mechanics. Notably, our results recover some wellknown entropic uncertainty relations for two arbitrary incompatible observables that demonstrate the uncertainties about the results of two measurements. This uncertainty relation might play a critical role in the foundations of quantum theory.

The uncertainty principle is a special feature of quantum mechanics. Historically, the first uncertainty relation formulated by Heisenberg ${ }^{1}$ who showed that one cannot predict the results with arbitrary precision for two incompatible observables concurrently, such as measurements of position $x$ and momentum $p$ of a particle ${ }^{2}$. Following, Robertson ${ }^{3}$ proposed an inequality in terms of a standard deviation in respect to a pair of incompatible observables for the systemic state. Nevertheless, Robertson's uncertainty bound is state-dependent and so will be trivial for some states. In order to eliminate this defect, Deutsch ${ }^{4}$ proposed to utilise the Shannon entropy as a proper criterion of uncertainty and introduced the so-called entropic uncertainty relation (EUR). Moreover, Kraus ${ }^{5}$ improved Deutsch's uncertainty bound, and later Maassen and Uffink ${ }^{6}$ proved it as

$$
H(Q)+H(R) \geq-\log _{2}(c),
$$

where $H(Q)=-\sum_{i} q_{i} \log _{2} q_{i}$ and $H(R)=-\sum_{j} r_{j} \log _{2} r_{j}$ are the Shannon entropy of the probabilities of measurement results of the incompatible observables $Q$ and $R$, respectively. The complementarity $c=\max _{i, j}\left\{\left|\left\langle q_{i} \mid r_{j}\right\rangle\right|^{2}\right\}$ is the maximal overlap of $Q$ and $R$ with $\left|q_{i}\right\rangle$ and $\left|r_{j}\right\rangle$ being the eigenstates of $Q$ and $R$, respectively. Compared with Robertson's uncertainty bound, the bound of EUR (1) depends only on the complementarity of the two observables, avoiding the deficiency of state dependence. Noteworthily, a recent study has shown that the variance-based and entropy-like uncertainty relations are mutually equivalent ${ }^{7}$.

After that, a striking result of the uncertainty principle is to study the effect of a quantum memory that is achievable with current technology. Berta et al. ${ }^{8}$ revealed that the entropic uncertainty can be decreased with the help of memory particle which might be correlated with the measured system. They demonstrated a new uncertainty relation, which can be called quantum-memory-assisted entropic uncertainty relation (QMA-EUR). This relation can be described by the interesting uncertainty game between two legitimate players, Alice and Bob. At the beginning of the game, Bob has a pair of correlated particles, $A$ and $B$, and sends particle $A$ to Alice, with $B$ as a memory particle. In the next step, she carries out a measurement on her quantum system by choosing one of the observables $Q$ and $R$. Afterwards, she announces to Bob her choice of the measurement. Finally, Bob's task is to predict Alice's measurement outcome. During this game, the uncertainty via von-Neumann entropies can be written as

$$
S(Q \mid B)+S(R \mid B) \geq-\log _{2}(c)+S(A \mid B),
$$

where $S(A \mid B)=S\left(\rho_{A B}\right)-S\left(\rho_{B}\right)$ is the conditional von-Neumann entropy of $\rho_{A B}$ with $\rho_{B}=\operatorname{tr}_{A}\left(\rho_{A B}\right)$ and $S(\rho)=-\operatorname{tr}\left(\rho \log _{2} \rho\right)$ is the von-Neumann entropy. Also, $S(\mathcal{O} \mid B)=S\left(\rho_{\mathcal{O} B}\right)-S\left(\rho_{B}\right)$ with $\mathcal{O} \in\{Q, R\}$ is the conditional von-Neumann entropy of the post-measurement state after the quantum system $A$ is measured, $\rho_{Q B}=\sum_{i}\left(\left|q_{i}\right\rangle_{A}\left\langle q_{i}\right| \otimes \mathbb{I}_{B}\right) \rho_{A B}\left(\left|q_{i}\right\rangle_{A}\left\langle q_{i}\right| \otimes \mathbb{I}_{B}\right)$, likewise for $\rho_{R B}$, and $\mathbb{I}_{B}$ being an identity operator in Hilbert space of $B$.

In the literature, substantial efforts have been made to improve Berta et al's bound ${ }^{9-17}$. To be explicit, Pati et al. ${ }^{9}$ improved Berta et al's bound by a term added to the right-hand side of inequality (2). Indeed, Pati et al.'s bound is tighter than Berta et al.'s bound if the quantum discord is larger than the classical correlation. Then, Pramanik et al..$^{10}$ obtained a new entropic uncertainty relation based on fine graining, which led to an ultimate limit on the accuracy achievable in measurements made on two incompatible observables in the presence of

${ }^{1}$ Faculty of Physics, Semnan University, P.O.Box 35195-363, Semnan, Iran. 2Saeed's Quantum Information Group, P.O.Box 19395-0560, Tehran, Iran. ${ }^{3}$ Department of Physics, Salman Farsi University of Kazerun, Kazerun, Iran. ${ }^{4}$ Faculty of Physics, Urmia University of Technology, Urmia, Iran. ${ }^{\varpi}$ email: saeed@ssqig.com 
quantum memory. Moreover, Coles and Piani ${ }^{11}$ reported a strong bound by considering the second largest value of the overlap. Zhang et al. ${ }^{12}$ presented tighter bounds on both entropic uncertainty and information exclusion relations for multiple measurements in the presence of quantum memory. In Ref. ${ }^{13}$, Pramanik et al. derived a new form of the uncertainty relation through extractable classical information. In 2016, Xiao et al. ${ }^{14}$ tightened Berta et al.'s bound based on Coles and Piani's remarkable bound. Later, Chen et al. ${ }^{15}$ improved the lower bounds for the entropic uncertainty relations via polynomial functions. Besides, Huang et al. ${ }^{16}$ presented a Holevo bound for QMA-EUR, where the difference between the entropic uncertainties and the new bound is always a fixed value. More recently, $\mathrm{Li}$ and Qiao ${ }^{17}$ proposed a method to decrease the local uncertainty. In this remarkable study a new kind of uncertainty relation based on conditional majorization ${ }^{18-20}$ has been formulated, which can be calculated for any number of observables. According to this new class of uncertainty relation and in the presence of quantum memory, one can get lower bounds in comparison to the conditional entropic uncertainty relation. On the other hand, continuing progress has been done by some groups from an experimental viewpoint ${ }^{21-31}$. Furthermore, a promising effort was made by Adabi et al. ${ }^{32}$ to tighten the Berta et al.'s bound, which is expressed as

$$
S(Q \mid B)+S(R \mid B) \geq-\log _{2}(c)+S(A \mid B)+\max \{0, \delta\},
$$

where $\delta=I(A: B)-[I(Q: B)+I(R: B)], I(A: B)=S\left(\rho_{A}\right)+S\left(\rho_{B}\right)-S\left(\rho_{A B}\right)$ is mutual information between Alice and Bob, and $I(\mathcal{O}: B)=S\left(\rho_{B}\right)-\sum_{i} p_{i} S\left(\rho_{B \mid i}\right)$ is Holevo quantity. Note that, $\rho_{B \mid i}=\operatorname{tr}_{A}\left(\Pi_{i}^{A} \rho_{A B} \Pi_{i}^{A}\right) / p_{i}$ is the post-measurement state of Bob after measuring of observable $\mathcal{O}$ by Alice and $p_{i}=\operatorname{tr}_{A B}\left(\Pi_{i}^{A} \rho_{A B} \Pi_{i}^{A}\right)$ is the probability of $i$ th outcome.

Interestingly, the bipartite QMA-EUR has been the topic of many works in recent years ${ }^{33-48}$ (see Refs. ${ }^{49}$ and $^{50}$ for detailed reviews on bipartite QMA-EUR). However, Renes and Boileau ${ }^{51}$ showed that the bipartite QMA-EUR could be generalized to the tripartite case where two particles $B$ and $C$ are considered as the quantum memories. It is shown that the tripartite QMA-EUR can be written as

$$
S(Q \mid B)+S(R \mid C) \geq-\log _{2}(c) .
$$

In this scenario, Alice, Bob, and Charlie share a tripartite quantum state $\rho_{A B C}$ and then Alice carries out one of two observables ( $Q$ and $R$ ) on her system. Briefly, if Alice measures $Q$, then Bob's task is to minimize his uncertainty about $Q$ and whenever she measures $R$, then Charlie's task is to minimize his uncertainty about $R$. Most recently, Ming et al. ${ }^{52}$ improved the tripartite uncertainty bound of the inequality (4) as

$$
S(Q \mid B)+S(R \mid C) \geq-\log _{2}(c)+\max \{0, \Delta\},
$$

where

$$
\Delta=-\log _{2}(c)+2 S(A)-[I(A: B)+I(A: C)]+[I(Q: B)+I(R: C)]-H(Q)-H(R) .
$$

Subsequently, Dolatkhah et al. ${ }^{53}$ introduced a new lower bound for tripartite QMA-EUR that is tighter than Ming et al.s bound. The new inequality can be derived by

$$
S(Q \mid B)+S(R \mid C) \geq-\log _{2}(c)+\frac{S(A \mid B)+S(A \mid C)}{2}+\max \left\{0, \delta^{\prime}\right\},
$$

with

$$
\delta^{\prime}=\frac{I(A: B)+I(A: C)}{2}-[I(Q: B)+I(R: C)] .
$$

Within the above, it is obvious that the studies only have been focused on the case of two measurements ( $Q$ and $R$ ). However, many attempts have been made to generalize the entropic uncertainty relations to more than two observables ${ }^{54-63}$. Here, one can refer to the result of Liu et al. ${ }^{55}$ that they considered Maassen and Uffink bound for multi-observable ( $>2$ ) with the state of the measured system $A \equiv \rho_{A}$ which is generally a mixed state, viz

$$
\sum_{m=1}^{N} H\left(\mathcal{O}_{m}\right) \geq-\log _{2}(b)+(N-1) S(A),
$$

in which

$$
b=\max _{i_{N}}\left\{\sum_{i_{2} \sim i_{N-1}} \max _{i_{1}}\left[c\left(u_{i_{1}}^{1}, u_{i_{2}}^{2}\right)\right] \prod_{m=2}^{N-1} c\left(u_{i_{m}}^{m}, u_{i_{m}+1}^{m+1}\right)\right\},
$$

where $c\left(u_{i_{1}}^{1}, u_{i_{2}}^{2}\right)=\left|\left\langle u_{i_{1}}^{1} \mid u_{i_{2}}^{2}\right\rangle\right|^{2}$ and $c\left(u_{i_{m}}^{m}, u_{i_{m+1}}^{m+1}\right)=\left|\left\langle u_{i_{m}}^{m} \mid u_{i_{m+1}}^{m+1}\right\rangle\right|^{2}$ with $\left|u_{i_{m}}^{m}\right\rangle$ which is the $i$ th eigenstate of $\mathcal{O}_{m}$. Furthermore, this inequality in the presence of quantum memory $B$ is converted to ${ }^{55}$

$$
\sum_{m=1}^{N} H\left(\mathcal{O}_{m} \mid B\right) \geq-\log _{2}(b)+(N-1) S(A \mid B) .
$$

Until now, the inequalities revealed only for bipartite, tripartite, and multi-measurement cases, while the case of multipartite systems remains unstudied. In this paper, we will present a novel entropic uncertainty with quantum memory for multipartite systems where the memory is split into several parts. Herein, we highlight this relation 
for its key role in quantum theory and potential wide applications, as well as expect that this uncertainty relation can be demonstrated in various physical systems.

\section{Generalization of QMA-EUR}

The following theorem reveals how to obtain the QMA-EUR for multipartite systems.

Theorem 1 By considering the measured system state $A \equiv \rho_{A}$ that is generally mixed, the following multipartite uncertainty relation holds for any state $\rho_{A m}(m=1,2, \ldots, N)$

$$
\sum_{m=1}^{N} S\left(\mathcal{O}_{m} \mid P_{m}\right) \geq-\log _{2}(b)+\frac{N-1}{N} \sum_{m=1}^{N} S\left(A \mid P_{m}\right)+\max \{0, \kappa\}
$$

where

$$
\kappa=\frac{N-1}{N} \sum_{m=1}^{N} I\left(A: P_{m}\right)-\sum_{m=1}^{N} I\left(\mathcal{O}_{m}: P_{m}\right)
$$

with $\mathcal{O}_{m}$ and $P_{m}$ which are the different incompatible observables and the memory particles for mth measurement, respectively. In this scenario, Alice and the others share a multipartite quantum state $\rho_{\text {Am }}$ and then Alice carries out one of the observables $\left(\mathcal{O}_{m}, m=1,2, \ldots, N\right)$ on her system. If Alice measures $\mathcal{O}_{1}$, then $P_{1}$ has the task to minimize his uncertainty about $\mathcal{O}_{1}$. If she measures $\mathcal{O}_{2}$, then $P_{2}$ has the task to minimize his uncertainty about $\mathcal{O}_{2}$. Generally, whenever Alice measures $\mathcal{O}_{m}$, then $P_{m}$ has the task to minimize his uncertainty about $\mathcal{O}_{m}$.

Proof Regarding $S(\mathcal{O} \mid P)=H(\mathcal{O})-I(\mathcal{O}: P)$, the left-hand side of inequality (12) can be rewritten as

$$
\begin{aligned}
\sum_{m=1}^{N} S\left(\mathcal{O}_{m} \mid P_{m}\right) & =\sum_{m=1}^{N} H\left(\mathcal{O}_{m}\right)-\sum_{m=1}^{N} I\left(\mathcal{O}_{m}: P_{m}\right) \\
& \geq-\log _{2}(b)+(N-1) S(A)-\sum_{m=1}^{N} I\left(\mathcal{O}_{m}: P_{m}\right) \\
& =-\log _{2}(b)+(N-1)\left[S\left(A \mid P_{1}\right)+I\left(A: P_{1}\right)\right]-\sum_{m=1}^{N} I\left(\mathcal{O}_{m}: P_{m}\right),
\end{aligned}
$$

where the inequality follows from Liu et al.s result for $N$ measurements (9) and the last equation comes from the identity $S(A)=S\left(A \mid P_{1}\right)+I\left(A: P_{1}\right)$. By using $S(A)=S\left(A \mid P_{2}\right)+I\left(A: P_{2}\right)$ we have

$$
\sum_{m=1}^{N} S\left(\mathcal{O}_{m} \mid P_{m}\right) \geq-\log _{2}(b)+(N-1)\left[S\left(A \mid P_{2}\right)+I\left(A: P_{2}\right)\right]-\sum_{m=1}^{N} I\left(\mathcal{O}_{m}: P_{m}\right) .
$$

In general, for $m=1,2, \ldots, N$ by substituting the identity $S(A)=S\left(A \mid P_{m}\right)+I\left(A: P_{m}\right)$ into (14), one arrives at

$$
\sum_{m=1}^{N} S\left(\mathcal{O}_{m} \mid P_{m}\right) \geq-\log _{2}(b)+\frac{N-1}{N} \sum_{m=1}^{N} S\left(A \mid P_{m}\right)+\frac{N-1}{N} \sum_{m=1}^{N} I\left(A: P_{m}\right)-\sum_{m=1}^{N} I\left(\mathcal{O}_{m}: P_{m}\right),
$$

which can be rewritten as the desired outcome (12).

Corollary 1 If the prepared state is a bipartite state, our uncertainty relation will recover Adabi et al.'s result.

Proof For any bipartite state, we consider two observables $\left(\mathcal{O}_{1}=Q\right.$ and $\left.\mathcal{O}_{2}=R\right)$ and $P_{1}=P_{2}=B$. Therefore, from Eq. (12) we obtain the Eq. (3) with the complementarity $b=c$ and $\kappa=\delta$.

Corollary 2 If the prepared state is a tripartite state, our uncertainty relation recover Dolatkhah et al.'s result.

Proof For any tripartite state, we consider two observables $\left(\mathcal{O}_{1}=Q\right.$ and $\left.\mathcal{O}_{2}=R\right)$ and $P_{1}=B$ and $P_{2}=C$. So, we restore the Eq. (7) with the complementarity $b=c$ and $\kappa=\delta^{\prime}$.

\section{Example: four-partite QMA-EUR}

Let $\mathcal{O}_{1}, \mathcal{O}_{2}$, and $\mathcal{O}_{3}$ be three incompatible observables for a four-partite system $\rho_{A B C D}$ which is generally a mixed state. The following four-partite uncertainty relation holds (with $P_{1}=B, P_{2}=C$, and $P_{3}=D$ )

$$
S\left(\mathcal{O}_{1} \mid B\right)+S\left(\mathcal{O}_{2} \mid C\right)+S\left(\mathcal{O}_{3} \mid D\right) \geq-\log _{2}\left(b^{\prime}\right)+\frac{2}{3}[S(A \mid B)+S(A \mid C)+S(A \mid D)]+\max \left\{0, \kappa^{\prime}\right\}
$$

where 


$$
\kappa^{\prime}=\frac{2}{3}[I(A: B)+I(A: C)+I(A: D)]-I\left(\mathcal{O}_{1}: B\right)-I\left(\mathcal{O}_{2}: C\right)-I\left(\mathcal{O}_{3}: D\right)
$$

and

$$
b^{\prime}=\max _{k}\left\{\sum_{j} \max _{i}\left[\left|\left\langle u_{i}^{1} \mid u_{j}^{2}\right\rangle\right|^{2}\right]\left|\left\langle u_{j}^{2} \mid u_{k}^{3}\right\rangle\right|^{2}\right\},
$$

with $\left|u_{i}^{1}\right\rangle,\left|u_{j}^{2}\right\rangle$ and $\left|u_{k}^{3}\right\rangle$ being the eigenstates of the three observables $\mathcal{O}_{1}, \mathcal{O}_{2}$ and $\mathcal{O}_{3}$, respectively.

It is worth noting that the memory has been split into three parts. In this situation, after preparing a fourpartite state $\rho_{A B C D}$, particle $A$ is sent to Alice, $B$ to Bob, $C$ to Charlie, and $D$ to David. Now, Alice carries out one of the three observables $\left(\mathcal{O}_{m}, m=1,2,3\right)$ on her system. Then, Alice informs Bob, Charlie, and David of her measurement choice. If Alice measures $\mathcal{O}_{1}$, then Bob's task is to minimize his uncertainty about $\mathcal{O}_{1}$. If she measures $\mathcal{O}_{2}$, then Charlie's task is to minimize his uncertainty about $\mathcal{O}_{2}$. Finally, if she measures $\mathcal{O}_{3}$, then David's task is to minimize his uncertainty about $\mathrm{O}_{3}$.

\section{Conclusion}

In summary, we have presented a generalized uncertainty relation with quantum memory for multipartite systems and obtained a new QMA-EUR for four-partite quantum systems. This generalized entropic uncertainty depends on the conditional von-Neumann entropies, Holevo quantities, and the mutual information. We expect that the inequality will bring on more potential applications in quantum information and communication, e.g., entanglement detection ${ }^{64}$, multipartite entanglement-structure detection ${ }^{65}$, witnessing multipartite entanglement ${ }^{66}$, detection of genuine multipartite entanglement in multipartite systems ${ }^{67}$, exploring the efficient multipartite entanglement criteria ${ }^{68-70}$, analyzing the monogamy and polygamy relations of multipartite quantum states ${ }^{71,72}$, and so on. It means that our multipartite uncertainty relation will have significant applications in entanglement detection and precision measurements. In a forthcoming paper, one may motivate to extend the results to get an uncertainty relation for multipartite systems based on conditional majorization in comparison to recent study ${ }^{17}$.

Received: 23 March 2021; Accepted: 21 June 2021

Published online: 02 July 2021

\section{References}

1. Heisenberg, W. Über den anschaulichen Inhalt der quantentheoretischen Kinematik und Mechanik. Z. Phys. 43, 172 (1927).

2. Kennard, E. H. Zur Quantenmechanik einfacher Bewegungstypen. Z. Phys. 44, 326 (1927).

3. Robertson, H. P. The uncertainty principle. Phys. Rev. 34, 163 (1929).

4. Deutsch, D. Uncertainty in quantum measurements. Phys. Rev. Lett. 50, 631 (1983).

5. Kraus, K. Complementary observables and uncertainty relations. Phys. Rev. D 35, 3070 (1987).

6. Maassen, H. \& Uffink, J. B. M. Generalized entropic uncertainty relations. Phys. Rev. Lett. 60, 1103 (1988).

7. Li, J. L. \& Qiao, C. F. Equivalence theorem of uncertainty relations. J. Phys. A Math. Theor. 50, 03LT01 (2017).

8. Berta, M., Christandl, M., Colbeck, R., Renes, J. M. \& Renner, R. The uncertainty principle in the presence of quantum memory. Nat. Phys. 6, 659 (2010).

9. Pati, A. K., Wilde, M. M., Usha Devi, A. R., Rajagopal, A. K. \& Sudha,. Quantum discord and classical correlation can tighten the uncertainty principle in the presence of quantum memory. Phys. Rev. A 86, 042105 (2012).

10. Pramanik, T., Chowdhury, P. \& Majumdar, A. S. Fine-grained lower limit of entropic uncertainty in the presence of quantum memory. Phys. Rev. Lett. 110, 020402 (2013).

11. Coles, P. J. \& Piani, M. Improved entropic uncertainty relations and information exclusion relations. Phys. Rev. A 89, 022112 (2014).

12. Zhang, J., Zhang, Y. \& Yu, C. S. Entropic uncertainty relation and information exclusion relation for multiple measurements in the presence of quantum memory. Sci. Rep. 5, 11701 (2015).

13. Pramanik, T., Mal, S. \& Majumdar, A. S. Lower bound of quantum uncertainty from extractable classical information. Quantum Inf. Process. 15, $981(2016)$.

14. Xiao, Y., Jing, N., Fei, S. . M. \& Li-Jost, X. Improved uncertainty relation in the presence of quantum memory. J. Phys. A Math. Theor. 9, 49LT01 (2016).

15. Chen, Z., Ma, Z., Xiao, Y. \& Fei, S. M. Improved quantum entropic uncertainty relations. Phys. Rev. A 98, 042305 (2018).

16. Huang, J. L., Gan, W. C., Xiao, Y. L., Shu, F. W. \& Yung, M. H. Holevo bound of entropic uncertainty in Schwarzschild spacetime. Eur. Phys. J. C 78, 545 (2018).

17. Li, J. L. \& Qiao, C. F. An optimal measurement strategy to beat the quantum uncertainty in correlated system. Adv. Quantum Technol. 3, 2000039 (2020).

18. Li, J. L. \& Qiao, C. F. The optimal uncertainty relation. Ann. Phys. (Berlin) 531, 1900143 (2019).

19. Rudnicki, Ł, Puchala, Z. \& Życzkowski, K. Strong majorization entropic uncertainty relations. Phys. Rev. A 89, 052115 (2014).

20. Rudnicki, Ł. Majorization approach to entropic uncertainty relations for coarse-grained observables. Phys. Rev. A 91, 032123 (2015).

21. Prevedel, R., Hamel, D. R., Colbeck, R., Fisher, K. \& Resch, K. J. Experimental investigation of the uncertainty principle in the presence of quantum memory and its application to witnessing entanglement. Nat. Phys. 7, 757 (2011).

22. Li, C. F., Xu, J. S., Xu, X. Y., Li, K. \& Guo, G. C. Experimental investigation of the entanglement-assisted entropic uncertainty principle. Nat. Phys. 7, 752 (2011).

23. Ma, W. C. et al. Experimental test of Heisenberg's measurement uncertainty relation based on statistical distances. Phys. Rev. Lett. 116, 160405 (2016).

24. Chen, Z. X. et al. Experimental investigation of multi-observable uncertainty relations. Phys. Rev. A 96, 062123 (2017),

25. Lv, W. M. et al. Experimental test of the trade-off relation for quantum coherence. Phys. Rev. A 98, 062337 (2018).

26. Wang, H. Y. et al. Uncertainty equality with quantum memory and its experimental verification. npj Quantum Inf. 5, 39 (2019).

27. Chen, Z. X., Wang, H., Li, J. L., Song, Q. C. \& Qiao, C. F. Tight N-observable uncertainty relations and their experimental demonstrations. Sci. Rep. 9, 5687 (2019).

28. Lv, W. M. et al. Experimental test of fine-grained entropic uncertainty relation in the presence of quantum memory. Sci. Rep. 9, 8748 (2019). 
29. Wang, H., Li, J. L., Wang, S., Song, Q. C. \& Qiao, C. F. Experimental investigation of the uncertainty relations with coherent light. Quantum Inf. Process. 19, 38 (2020).

30. Ding, Z. Y. et al. Experimental investigation of entropic uncertainty relations and coherence uncertainty relations. Phys. Rev. A 101, 032101 (2020).

31. Yang, H. et al. Experimental certification of the steering criterion based on a general entropic uncertainty relation. Phys. Rev. A 101, $022324(2020)$.

32. Adabi, F., Salimi, S. \& Haseli, S. Tightening the entropic uncertainty bound in the presence of quantum memory. Phys. Rev. A 93, 062123 (2016).

33. Coles, P. J., Colbeck, R., Yu, L. \& Zwolak, M. Uncertainty relations from simple entropic properties. Phys. Rev. Lett. 108, 210405 (2012).

34. Hu, M. L. \& Fan, H. Upper bound and shareability of quantum discord based on entropic uncertainty relations. Phys. Rev. A 88, 014105 (2013).

35. Wang, D. et al. Quantum-memory-assisted entropic uncertainty relation in a Heisenberg XYZ chain with an inhomogeneous magnetic field. Laser Phys. Lett. 14, 065203 (2017).

36. Wang, D., Ming, F., Huang, A. J., Sun, W. Y. \& Ye, L. Entropic uncertainty for spin-1/2 XXX chains in the presence of inhomogeneous magnetic fields and its steering via weak measurement reversals. Laser Phys. Lett. 14, 095204 (2017).

37. Wang, D. et al. Exploration of quantum-memory-assisted entropic uncertainty relations in a noninertial frame. Laser Phys. Lett. 14, 055205 (2017).

38. Pourkarimi, M. R. Quantum correlations and entropic uncertainty relation in a three-qubit spin chain under the effect of magnetic field and DM interaction. Int. J. Quantum Inform. 16, 1850057 (2018).

39. Pourkarimi, M. R. Time evolution of quantum-memory-assisted entropic uncertainty relation and quantum correlations under dissipative environment. Int. J. Quantum Inform. 17, 1950008 (2019).

40. Haddadi, S., Pourkarimi, M. R., Akhound, A. \& Ghominejad, M. Quantum correlations and quantum-memory-assisted entropic uncertainty relation in two kinds of spin squeezing models. Laser Phys. Lett. 16, 095202 (2019).

41. Pourkarimi, M. R. \& Haddadi, S. Quantum-memory-assisted entropic uncertainty, teleportation, and quantum discord under decohering environments. Laser Phys. Lett. 17, 025206 (2020).

42. Haseli, S., Dolatkhah, H., Salimi, S. \& Khorashad, A. S. Controlling the entropic uncertainty lower bound in two-qubit systems under decoherence. Laser Phys. Lett. 16, 045207 (2019).

43. Haddadi, S., Ghominejad, M., Akhound, A. \& Pourkarimi, M. R. Exploring entropic uncertainty relation and dense coding capacity in a two-qubit X-state. Laser Phys. Lett. 17, 095205 (2020).

44. Haseli, S., Haddadi, S. \& Pourkarimi, M. R. Entropic uncertainty lower bound for a two-qubit system coupled to a spin chain with Dzyaloshinskii-Moriya interaction. Opt. Quant. Electron. 52, 465 (2020).

45. Pourkarimi, M. R., Haddadi, S. \& Haseli, S. Exploration of entropic uncertainty bound in a symmetric multi-qubit system under noisy channels. Phys. Scr. 96, 015101 (2021).

46. Haseli, S., Haddadi, S. \& Pourkarimi, M. R. Probing the entropic uncertainty bound and quantum correlations in a quantum dot system. Laser Phys. 31, 055203 (2021).

47. Haddadi, S., Haseli, S., Pourkarimi, M. R. \& Mirmasoudi, F. Analyzing entropic uncertainty bound in two qubits coupled to a spin environment. Phys. Scr. 96, 075104 (2021).

48. Li, J. L. \& Qiao, C. F. The generalized uncertainty principle. Ann. Phys. (Berlin) 533, 2000335 (2021).

49. Coles, P. J., Berta, M., Tomamichel, M. \& Wehner, S. Entropic uncertainty relations and their applications. Rev. Mod. Phys. 89, 015002 (2017).

50. Wang, D., Ming, F., Hu, M. L. \& Ye, L. Quantum-memory-assisted entropic uncertainty relations. Ann. Phys. (Berlin) 531, 1900124 (2019).

51. Renes, J. M. \& Boileau, J. C. Conjectured strong complementary information tradeoff. Phys. Rev. Lett. 103, 020402 (2009).

52. Ming, F. et al. Improved tripartite uncertainty relation with quantum memory. Phys. Rev. A 102, 012206 (2020).

53. Dolatkhah, H., Haseli, S., Salimi, S. \& Khorashad, A. S. Tightening the tripartite quantum-memory-assisted entropic uncertainty relation. Phys. Rev. A 102, 052227 (2020).

54. Wehner, S. \& Winter, A. Entropic uncertainty relations-a survey. New J. Phys. 12, 025009 (2010).

55. Liu, S., Mu, L. Z. \& Fan, H. Entropic uncertainty relations for multiple measurements. Phys. Rev. A 91, 042133 (2015).

56. Li, J. L. \& Qiao, C. F. Reformulating the quantum uncertainty relation. Sci. Rep. 5, 12708 (2015).

57. Chen, B. \& Fei, S. M. Sum uncertainty relations for arbitrary N incompatible observables. Sci. Rep. 5, 14238 (2015).

58. Song, Q. C. \& Qiao, C. F. Stronger Schrödinger-like uncertainty relations. Phys. Lett. A 380, 2925 (2016).

59. Xiao, Y., Jing, N., Li-Jost, X. \& Fei, S. M. Weighted uncertainty relations. Sci. Rep. 6, 23201 (2016).

60. Xiao, Y. et al. Strong entropic uncertainty relations for multiple measurements. Phys. Rev. A 93, 042125 (2016).

61. Qin, H. H., Fei, S. M. \& Li-Jost, X. Multi-observable uncertainty relations in product form of variances. Sci. Rep. 6, 31192 (2016).

62. Song, Q. C., Li, J. L., Peng, G. X. \& Qiao, C. F. A stronger multi-observable uncertainty relation. Sci. Rep. 7, 44764 (2017).

63. Schwonnek, R., Dammeier, L. \& Werner, R. F. State-independent uncertainty relations and entanglement detection in noisy systems. Phys. Rev. Lett. 119, 170404 (2017).

64. Gühne, O. \& Tóth, G. Entanglement detection. Phys. Rep. 474, 1 (2009).

65. Zhou, Y., Zhao, Q., Yuan, X. \& Ma, X. Detecting multipartite entanglement structure with minimal resources. npj Quantum Inf. 5, $83(2019)$.

66. Girolami, D. \& Yadin, B. Witnessing multipartite entanglement by detecting asymmetry. Entropy 19, 124 (2017).

67. Zhao, J. Y., Zhao, H., Jing, N. \& Fei, S. M. Detection of genuine multipartite entanglement in multipartite systems. Int. J. Theor. Phys. 58, 3181 (2019).

68. Szalay, S. Multipartite entanglement measures. Phys. Rev. A 92, 042329 (2015).

69. Haddadi, S. \& Bohloul, M. A brief overview of bipartite and multipartite entanglement measures. Int. J. Theor. Phys. 57, 3912 (2018)

70. Guo, Y. \& Zhang, L. Multipartite entanglement measure and complete monogamy relation. Phys. Rev. A 101, 032301 (2020).

71. Jin, Z. X., Fei, S. M. \& Qiao, C. F. Polygamy relations of multipartite systems. Quantum Inf. Process. 18, 105 (2019).

72. Jin, Z. X., Fei, S. M. \& Qiao, C. F. Complementary quantum correlations among multipartite systems. Quantum Inf. Process. 19, $101(2020)$.

\section{Author contributions}

S. Haddadi has put forward the main idea. S. Haddadi, M. R. Pourkarimi, and S. Haseli all contributed to the development and completion of the idea, performing the calculations, analyzing the results, discussions and writing the manuscript. The final draft of the manuscript was revised by S. Haddadi and reviewed by all authors.

\section{Competing interests}

The authors declare no competing interests. 


\section{Additional information}

Correspondence and requests for materials should be addressed to S.H.

Reprints and permissions information is available at www.nature.com/reprints.

Publisher's note Springer Nature remains neutral with regard to jurisdictional claims in published maps and institutional affiliations.

(c) (1) Open Access This article is licensed under a Creative Commons Attribution 4.0 International License, which permits use, sharing, adaptation, distribution and reproduction in any medium or format, as long as you give appropriate credit to the original author(s) and the source, provide a link to the Creative Commons licence, and indicate if changes were made. The images or other third party material in this article are included in the article's Creative Commons licence, unless indicated otherwise in a credit line to the material. If material is not included in the article's Creative Commons licence and your intended use is not permitted by statutory regulation or exceeds the permitted use, you will need to obtain permission directly from the copyright holder. To view a copy of this licence, visit http://creativecommons.org/licenses/by/4.0/.

(C) The Author(s) 2021 